\title{
Nonadiabatic quantum dynamics without potential energy surfaces
}

\author{
Guillermo Albareda, ${ }^{1,2, *}$ Aaron Kelly, ${ }^{1,3, \dagger}$ and Angel Rubio ${ }^{1,4, \dagger}$ \\ ${ }^{1}$ Max Planck Institute for the Structure and Dynamics of Matter and Center for Free-Electron Laser Science, \\ Luruper Chaussee 149, 22761 Hamburg, Germany \\ ${ }^{2}$ Institute of Theoretical and Computational Chemistry, University of Barcelona, Martí $i$ Franquès 1-11, 08028 Barcelona, Spain \\ ${ }^{3}$ Department of Chemistry, Dalhousie University, Halifax, Canada B3H $4 R 2$ \\ ${ }^{4}$ Center for Computational Quantum Physics (CCQ), Flatiron Institute, 162 Fifth Avenue, New York, New York 10010, USA
}

(Received 5 June 2018; revised manuscript received 24 December 2018; published 22 February 2019)

\begin{abstract}
We present an ab initio algorithm for quantum dynamics simulations that reformulates the traditional "curse of dimensionality" that plagues all state-of-the-art techniques for solving the time-dependent Schrödinger equation. Using a stochastic wave-function ansatz that is based on a set of interacting single-particle conditional wave functions, we show that the difficulty of the problem becomes dominated by the number of trajectories needed to describe the process, rather than simply the number of degrees of freedom involved. This highly parallelizable technique achieves quantitative accuracy for situations in which mean-field theory drastically fails to capture qualitative aspects of the dynamics, such as quantum decoherence or the reduced nuclear probability density, using orders of magnitude fewer trajectories than a mean-field simulation. We illustrate the performance of this method for two fundamental nonequilibrium processes: a photoexcited proton-coupled electron transfer problem, and nonequilibrium dynamics in a cavity bound electron-photon system in the ultrastrong-coupling regime.
\end{abstract}

DOI: 10.1103/PhysRevMaterials.3.023803

\section{INTRODUCTION}

New computational tools are still needed to treat nonequilibrium many-body quantum dynamics problems in molecular and condensed phase systems. Pragmatically speaking, while exact solutions are out of reach for a broad range of systems, our main goal is to provide an alternative theoretical framework for generating simulation-based predictions of observable properties that are as accurate as possible, in a computationally feasible, $a b$ initio, manner.

Trajectory-based quantum dynamics methods provide one possible route toward this goal, and they offer the standard tradeoff between physical accuracy and computational cost [1-3]. Of these approaches, perhaps the most popular are Ehrenfest mean field theory [4] and Tully's surface hopping dynamics [5]. Both of these approaches can be simulated using an ensemble of uncorrelated trajectories. Reintroducing correlation, for example by using wave-packet methods [6-10], semiclassical techniques [11,12], the quantum-classical Liouville equation [13-15], linearizationbased approaches [16-19], or methods based on the exact factorization [20-22], allows for further accuracy at the expense of (often significant) additional computational effort. In practice, essentially all of these quantum dynamics methods are formulated using a discrete (truncated) Hilbert space representation for the electronic degrees of freedom. In this picture, the Born-Oppenheimer (BO) approximation naturally emerges as classical nuclear dynamics on the

\footnotetext{
*guillermo.albareda@mpsd.mpg.de

$\dagger$ aaron.kelly@dal.ca

†angel.rubio@mpsd.mpg.de
}

electronic ground-state potential energy surface (BOPES) [23], and nonadiabatic effects are introduced by including multiple electronic potential energy surfaces and nonadiabatic coupling terms (NACTs) [24].

An alternative to this approach is to use the (real-space) position representation for the electrons. This allows one to go beyond the $\mathrm{BO}$ picture without the need to explicitly calculate several BOPESs and NACTs [25]. This is an attractive feature from a computational point of view, as these quantities may be demanding to obtain from $a b$ initio electronic structure calculations. The conditional wave-function approach (CWF) can be formulated in this picture; it is an exact decomposition and recasting of the unitary time-evolution of a closed quantum system that yields a set of coupled, non-Hermitian, equations of motion [26]. Inspired by the trajectory-based approach to quantum dynamics of de Broglie and Bohm [27-30], the CWF approach allows one to describe the evolution of arbitrary subsets of the degrees of freedom in a system, on a formally exact level. In addition, this alternative formulation of the many-body quantum dynamics problem allows novel approximate schemes to be developed [31,32], providing a completely new perspective to deal with the long-standing problems of nonadiabatic dynamics in complex interacting systems.

In this paper, we report an approach for performing nonadiabatic quantum dynamics simulations using a set of timeevolving basis functions that are obtained from an approximation to the exact CWF equations of motion. This technique allows one to bypass the, typically necessary, computation of multiple BOPESs and NACTs, and potential subsequent diabatization procedures. Hence, this method offers an attractive route to calculate observables and time correlation functions without relying on the widely used concept of the BOPES. 


\section{THEORY}

We consider a closed system of interacting particles, and we separate the degrees of freedom into two arbitrary subsets. We also use the position representation for both sets; lowercase symbols will be used for the first subsystem, e.g., $\mathbf{r}=$ $\left\{\mathbf{r}_{1}, \ldots, \mathbf{r}_{n}\right\}$, and uppercase symbols $\mathbf{R}=\left\{\mathbf{R}_{1}, \ldots, \mathbf{R}_{N}\right\}$ for the second. This decomposition holds for an arbitrary number of subsets (up to the total number of degrees of freedom in the system), and it applies to both fermionic and bosonic many-body interacting systems. Here we choose $n$ and $N$ to represent the total number of degrees of freedom in each of the two subsystems.

The conditional wave function (CWF) approach can be developed starting from the full time-dependent Schrödinger equation (TDSE) in the position representation,

$$
i \hbar \frac{\partial}{\partial t} \Psi(\mathbf{r}, \mathbf{R}, t)=\hat{H}(\mathbf{r}, \mathbf{R}, t) \Psi(\mathbf{r}, \mathbf{R}, t) .
$$

The total Hamiltonian for the system is

$$
\hat{H}=\hat{T}_{1}(\mathbf{r})+\hat{T}_{2}(\mathbf{R})+W(\mathbf{r}, \mathbf{R}, t),
$$

where the kinetic energy operators for each species $j$ are $\hat{T}_{j}=$ $\frac{\hbar^{2}}{2 m_{j}}\left[i \nabla_{j}-\mathbf{A}_{j}(t)\right]^{2}$, and $m_{j}$ are their characteristic masses. The vector potential (in Coulomb gauge) due to an arbitrary external electromagnetic field, $\mathbf{A}_{j}(\mathbf{r}, \mathbf{R}, t)$, is also included. The full interacting potential energy of the system is $W(\mathbf{r}, \mathbf{R}, t)$.

The total wave function can be exactly decomposed in terms of the CWFs of either of the two subsystems,

$$
\begin{aligned}
\psi_{1}^{\alpha}(\mathbf{r}, t) & :=\int d \mathbf{R} \delta\left(\mathbf{R}^{\alpha}(t)-\mathbf{R}\right) \Psi(\mathbf{r}, \mathbf{R}, t), \\
\psi_{2}^{\alpha}(\mathbf{R}, t) & :=\int d \mathbf{r} \delta\left(\mathbf{r}^{\alpha}(t)-\mathbf{r}\right) \Psi(\mathbf{r}, \mathbf{R}, t),
\end{aligned}
$$

where the index $\alpha$ describes an ensemble of trajectories stochastically sampled from the initial probability density $|\Psi(\mathbf{r}, \mathbf{R}, 0)|^{2}$ (see the Supplemental Material in Ref. [33] for a detailed description of the numerical implementation of the ICWF method). Using these definitions in Eq. (1), one can show that the CWFs, $\psi_{1}^{\alpha}(t)$ and $\psi_{2}^{\alpha}(t)$, obey the following equations of motion:

$$
\begin{aligned}
i \hbar \frac{d \psi_{1}^{\alpha}}{d t} & =\left(\hat{T}_{1}(\mathbf{r})+W\left(\mathbf{r}, \mathbf{R}^{\alpha}, t\right)+\eta_{1}^{\alpha}(\mathbf{r}, t)\right) \psi_{1}^{\alpha}, \\
i \hbar \frac{d \psi_{2}^{\alpha}}{d t} & =\left(\hat{T}_{2}(\mathbf{R})+W\left(\mathbf{r}^{\alpha}, \mathbf{R}, t\right)+\eta_{2}^{\alpha}(\mathbf{R}, t)\right) \psi_{2}^{\alpha},
\end{aligned}
$$

where we have suppressed the explicit time-dependence of the coordinates, i.e., $\left\{\mathbf{r}^{\alpha}, \mathbf{R}^{\alpha}\right\} \equiv\left\{\mathbf{r}^{\alpha}(t), \mathbf{R}^{\alpha}(t)\right\}$. The complex potentials $\eta_{1}^{\alpha}(\mathbf{r}, t)$ and $\eta_{2}^{\alpha}(\mathbf{R}, t)$ are functionals of the full wave unction, and they are given in Refs. [25,26]. The conditional wave functions, (3) and (4), represent slices of the full wave function taken along the degrees of freedom of the two disjoint subsets (see, e.g., Fig. 2 in Ref. [25]). Each individual conditional wave function constitutes an open quantum system, whose time-evolution is nonunitary, due to the complex potentials. Notice also that, due to the fact that the two subsets of degrees of freedom $\mathbf{r}$ and $\mathbf{R}$ are treated on the same mathematical footing, the conditional decomposition in
Eqs. (3) and (4) links the number of trajectories used for both subspaces.

An exact solution to Eq. (1) can be constructed provided there is an ensemble of trajectories $\left\{\mathbf{r}^{\alpha}, \mathbf{R}^{\alpha}\right\}$ that explores the full support of $|\Psi(\mathbf{r}, \mathbf{R}, t)|^{2}$. For example, an ensemble of Bohmian trajectories defined through the conditional velocity fields [34,35] would fulfill such requirements. An approximate solution can be formulated [26] by expanding the complex functionals around the conditional coordinates, and then truncating such that $\eta_{1}^{\alpha}(\mathbf{r}, t)=f\left(\mathbf{R}^{\alpha}, t\right)$ and $\eta_{2}^{\alpha}(\mathbf{R}, t)=$ $g\left(\mathbf{r}^{\alpha}, t\right)$. In this limit, these potentials only engender a pure time-dependent phase that can be omitted, as the conditional velocity fields are invariant under such global phase transformations [26]. The resulting propagation scheme is thus restored to a Hermitian form, and this approximate version of the CWF formalism is referred to as the Hermitian-CWF approach [26].

The Hermitian-CWF propagation scheme recasts the full quantum time-propagator as a set of independent singlespecies propagators, which is clearly a major simplification of the full problem. Hence, this form of the conditional decomposition allows one to circumvent the problem of storing and propagating the full many-body wave function, whose size scales exponentially with the number of degrees of freedom.

In this paper, we consider the following ansatz for the full many-body wave function:

$$
\Psi(\mathbf{r}, \mathbf{R}, t)=\sum_{\alpha=1}^{M} C_{\alpha}(t) \psi_{1}^{\alpha}(\mathbf{r}, t) \psi_{2}^{\alpha}(\mathbf{R}, t) .
$$

The basis functions in this sum are chosen to be HermitianCWFs, and the upper limit of the sum, $M$, refers to the total number of stochastically sampled trajectories (which, as we will show below, can be kept to a very low number, making the present scheme computationally very efficient). Including interactions between the trajectories in the ensemble corrects the Hermitian-CWF evolution, through the set of complex time-dependent coefficients, $\mathbf{C}(t)=\left\{C_{1}(t), \ldots, C_{M}(t)\right\}$. The time evolution of these coefficients is obtained by inserting Eq. (7) into Eq. (1),

$$
i \hbar \mathbb{M} \dot{\mathbf{C}}(t)=\left(\mathbb{W}-\mathbb{W}_{1}-\mathbb{W}_{2}\right) \mathbf{C}(t),
$$

where the matrix elements of $\mathbb{M}, \mathbb{W}, \mathbb{W}_{1}$, and $\mathbb{W}_{2}$ are

$$
\begin{aligned}
\mathrm{M}_{\alpha^{\prime}, \alpha} & =\int d \mathbf{r} \psi_{1}^{\alpha^{\prime} *} \psi_{1}^{\alpha} \int d \mathbf{R} \psi_{2}^{\alpha^{\prime} *} \psi_{2}^{\alpha}, \\
\mathrm{W}_{\alpha^{\prime}, \alpha} & =\int d \mathbf{r} d \mathbf{R} \psi_{1}^{\alpha^{\prime} *} \psi_{1}^{\alpha} \psi_{2}^{\alpha^{\prime} *} \psi_{2}^{\alpha} W(\mathbf{r}, \mathbf{R}), \\
\mathrm{W}_{1}^{\alpha^{\prime}, \alpha} & =\int d \mathbf{r} \psi_{1}^{\alpha^{\prime} *} \psi_{1}^{\alpha} W\left(\mathbf{r}, \mathbf{R}^{\alpha}\right) \int d \mathbf{R} \psi_{2}^{\alpha^{\prime} *} \psi_{2}^{\alpha}, \\
\mathrm{W}_{2}^{\alpha^{\prime}, \alpha} & =\int d \mathbf{r} \psi_{1}^{\alpha^{\prime} *} \psi_{1}^{\alpha} \int d \mathbf{R} \psi_{2}^{\alpha^{\prime} *} \psi_{2}^{\alpha} W\left(\mathbf{r}^{\alpha}, \mathbf{R}\right) .
\end{aligned}
$$

Obtaining these matrix elements is straightforward and, except for (9b), they can be easily calculated from independent single-species integrals. Evaluating the matrix elements of $\mathbb{W}$, in principle, requires the reconstruction of the full (ansatz) wave function. This does not restrict the use of the method to cases in which the potential energy $W(\mathbf{r}, \mathbf{R})$ can be 
fit to a sum-of-products form, as in the multiconfigurational time-dependent Hartree method [36], for example, but it does pose a potential numerical challenge in the case of a large trajectory ensemble.

Once the coefficients $\mathbf{C}(t)$ are known, the velocity fields $\left\{\dot{\mathbf{r}}^{\alpha}, \dot{\mathbf{R}}^{\alpha}\right\}$ are then constructed according to the exact expressions for each subsystem:

$$
\begin{aligned}
\dot{\mathbf{r}}_{\xi}^{\alpha}(t) & =\operatorname{Im}\left[\frac{\left.\sum_{\alpha} C_{\alpha}(t) \psi_{2}^{\alpha}\left(\mathbf{R}^{\alpha}, t\right)\left[\nabla_{\xi} \psi_{1}^{\alpha}(\mathbf{r}, t)\right]\right|_{\mathbf{r}^{\alpha}(t)}}{m_{\xi} \sum_{\alpha} C_{\alpha}(t) \psi_{1}^{\alpha}\left(\mathbf{r}^{\alpha}, t\right) \psi_{2}^{\alpha}\left(\mathbf{R}^{\alpha}, t\right)}\right], \\
\dot{\mathbf{R}}_{v}^{\alpha}(t) & =\operatorname{Im}\left[\frac{\left.\sum_{\alpha} C_{\alpha}(t) \psi_{1}^{\alpha}\left(\mathbf{r}^{\alpha}, t\right)\left[\nabla_{v} \psi_{2}^{\alpha}(\mathbf{R}, t)\right]\right|_{\mathbf{R}^{\alpha}(t)}}{m_{v} \sum_{\alpha} C_{\alpha}(t) \psi_{1}^{\alpha}\left(\mathbf{r}^{\alpha}, t\right) \psi_{2}^{\alpha}\left(\mathbf{R}^{\alpha}, t\right)}\right] .
\end{aligned}
$$

The interacting-CWF method, described above, does not require the electronic BOPES or NACs as input, or for time propagation. This feature is potentially quite advantageous for treating processes that involve many quantum states or continua, as in light-induced dynamics or surface-scattering phenomena. In addition, the interacting-CWF propagation scheme avoids the computation of the nonlocal complex potentials, $\eta_{1}^{\alpha}(\mathbf{r}, t)$ and $\eta_{2}^{\alpha}(\mathbf{R}, t)$, as it is based on the Hermitian limit of the CWF equations of motion. Furthermore, there is minimal cross-talk between trajectories, which makes the algorithm computationally efficient in massively parallel architectures [33].

\section{RESULTS AND DISCUSSION}

\section{A. Photoinduced proton-coupled electron transfer reaction}

We first show interacting-CWF simulation results for a prototypical photoinduced proton-coupled electron transfer reaction, using the Shin-Metiu model [37]. The system comprises donor and acceptor ions that are fixed at a distance $L=19.0 a_{0}$, and a proton and an electron that are free to move in one dimension along the line connecting the donor-acceptor complex (see Fig. 1). This model is very flexible and, based on the parameter regime chosen, can give rise to a number of challenging situations in which electron-nuclear correlations play a crucial role in the dynamics.

The total Hamiltonian for the system is

$$
\hat{H}(r, R)=-\frac{1}{2 m} \frac{\partial^{2}}{\partial r^{2}}-\frac{1}{2 M} \frac{\partial^{2}}{\partial R^{2}}+\hat{W}(r, R),
$$

where $m$ is the electron mass and $M$ is the proton mass. The coordinates of the electron and the mobile ion are measured from the center of the two fixed ions, and they are labeled $r$ and $R$, respectively. The full electron-nuclear potential is

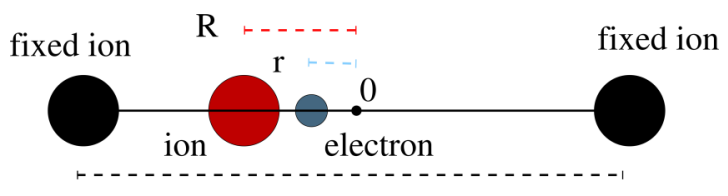

L

FIG. 1. Schematic representation of the Shin-Metiu model [37]. Two ions are fixed (in black) and a third one (in red) and an electron (in blue) are free to move in one dimension.

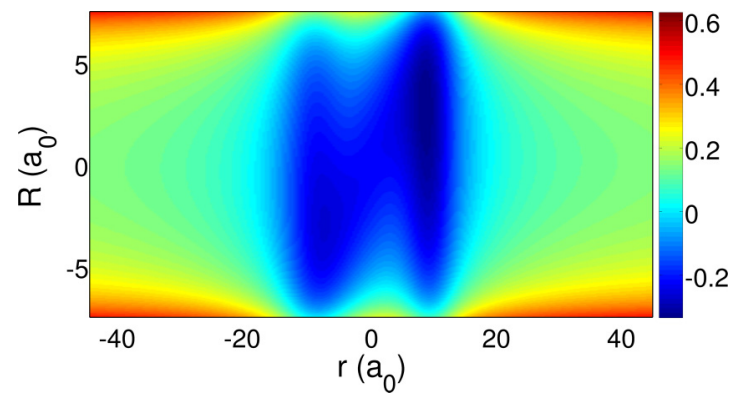

FIG. 2. Full electron-nuclear potential $\hat{W}(r, R)$ for the ShinMetiu model system.

depicted in Fig. 2 and reads

$$
\begin{aligned}
\hat{W}(r, R)= & \frac{1}{\left|\frac{L}{2}-R\right|}+\frac{1}{\left|\frac{L}{2}+R\right|}-\frac{\operatorname{erf}\left(\frac{|R-r|}{R_{f}}\right)}{|R-r|} \\
& -\frac{\operatorname{erf}\left(\frac{\left|r-\frac{L}{2}\right|}{R_{r}}\right)}{\left|r-\frac{L}{2}\right|}-\frac{\operatorname{erf}\left(\frac{\left|r+\frac{L}{2}\right|}{R_{l}}\right)}{\left|r+\frac{L}{2}\right|}
\end{aligned}
$$

where erf() represents the error function. The parameter regime studied in this model $\left(R_{f}=5 a_{0}, R_{l}=4 a_{0}\right.$, and $R_{r}=3.1 a_{0}$ ) was selected to compare with previous work on nonadiabatic relaxation in photoexcited electron-ion systems [26,38], and it was chosen such that the ground Born-Oppenheimer potential-energy surface (BOPES), $\epsilon_{\mathrm{BO}}^{(1)}$, is strongly coupled to the first excited adiabatic state, $\epsilon_{\mathrm{BO}}^{(2)}$, around $R_{\mathrm{ac}}=-2 a_{0}$. The coupling to the rest of the BOPESs is negligible (see Fig. 3).

We suppose the system to be initially uncorrelated, as if prepared by a short laser pulse, in the first excited BO electronic state, $\epsilon_{\mathrm{BO}}^{(2)}$, while the initial nuclear wave function is a Gaussian wave packet, with $\sigma=1 / \sqrt{2.85}$, centered on the equilibrium geometry of the ground $\mathrm{BO}$ state, at $R=$ $-4.0 a_{0}$. The time step used for integrating the TDSE is $2.4 \times$ $10^{-3}$ fs (or $0.1 a_{0}$ ). The fourth-order Runge-Kutta algorithm is used to propagate the CWF equations of motion and the corresponding trajectories. Propagating Eq. (8) requires the matrix inverse of $\mathbb{M}$ in Eq. (9a). However, this matrix can become ill-conditioned when different products of CWFs strongly overlap, or if the basis becomes overdetermined, for

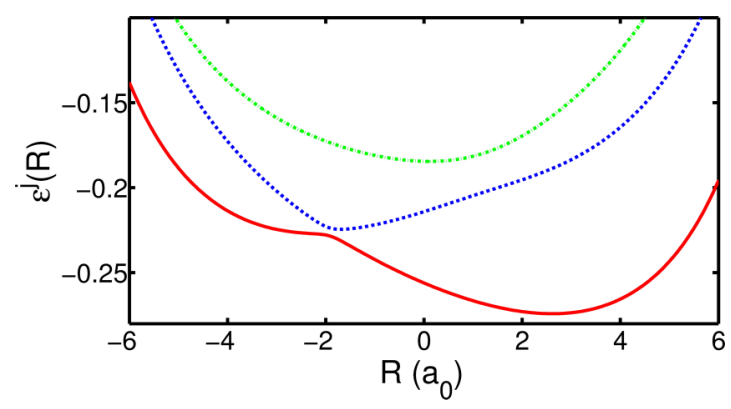

FIG. 3. BOPESs for the Shin-Metiu model system. Ground state, $\epsilon^{(1)}$, first excited state, $\epsilon^{(2)}$, and second excited state, $\epsilon^{(3)}$, BOPESs are shown, respectively, by a red solid line, a blue dashed line, and a green dotted line. 
example. We used the Moore-Penrose pseudoinversion method to ameliorate this numerical complication.

The short-time dynamics that proceeds from this initial condition involves a passage through an avoided crossing of two BOPESs, with further crossings occurring at later times as the system evolves. To characterize the dynamics, we monitor the BO electronic state populations, the reduced nuclear probability density, $\rho(\mathbf{R}, t)=$ $\int d \mathbf{r}|\Psi(\mathbf{r}, \mathbf{R}, t)|^{2}$, as well as an indicator of decoherence that is defined as the overlap integral of projected nuclear densities evolving on different BO electronic states, $D_{n m}(t)=$ $\int d \mathbf{R}\left|\chi^{(n)}(\mathbf{R}, t)\right|^{2}\left|\chi^{(m)}(\mathbf{R}, t)\right|^{2}$, where we have introduced the
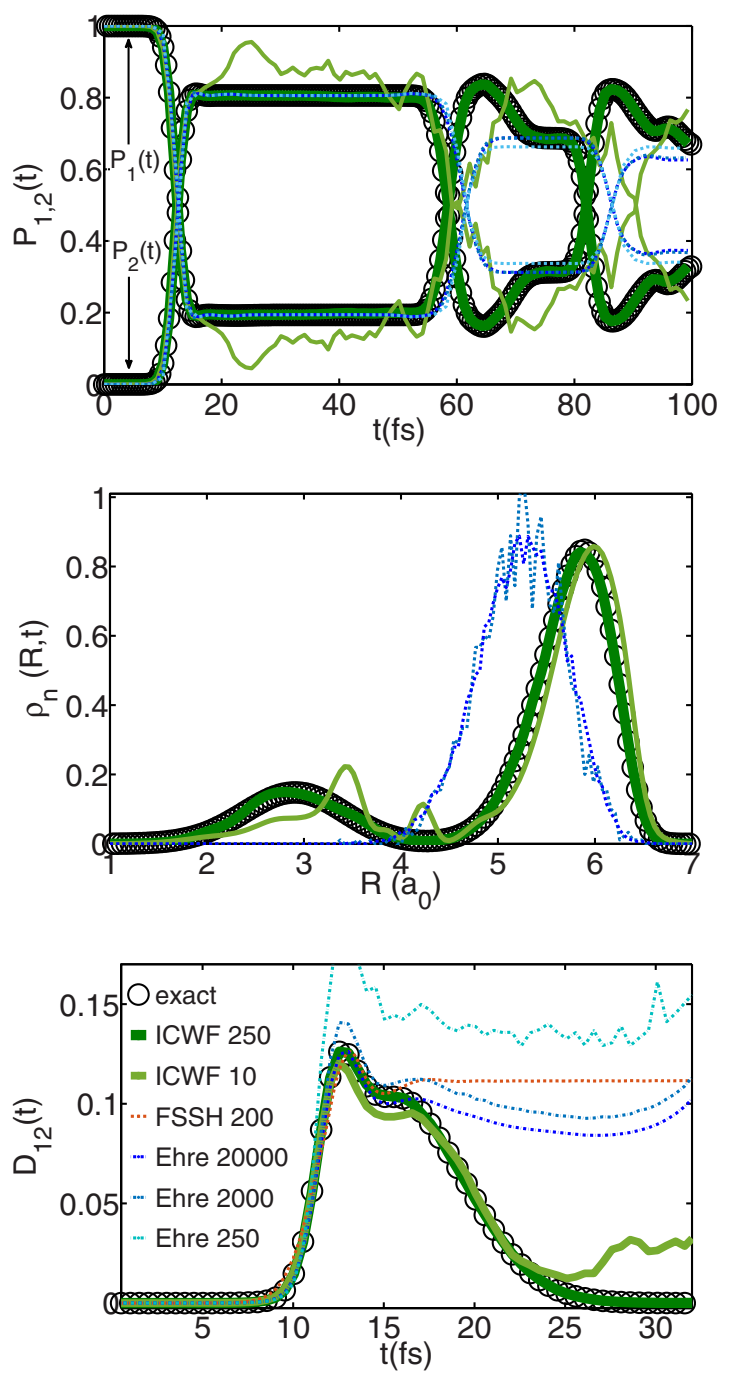

FIG. 4. Dynamics in the Shin-Metiu model for photoinduced proton-coupled electron transfer (see the text and the Supplemental Material in Ref. [33] for details). Exact results (black circles), interacting-CWF with $M=250$ (dark green line) and $M=10$ (light green line), and Ehrenfest mean field theory results with $M=20000$ (dark blue dashed line), $M=2000$ (blue dashed line), and $M=250$ (light blue dashed line). Top panel: evolution of the BO state populations $P_{1}(t)$ and $P_{2}(t)$. Middle panel: snapshot of the reduced nuclear coordinate density $\rho_{n}(R, t)$ at $t=31.84$ fs. Bottom panel: timedependent decoherence indicator $D_{12}(t)$; fewest-switches surfacehopping results with 200 trajectories (red dashed line). so-called Born-Huang expansion of the molecular wave function, $\Psi(\mathbf{r}, \mathbf{R}, t)=\sum_{n} \Phi_{\mathbf{R}}^{(n)}(\mathbf{r}, t) \chi^{(n)}(\mathbf{R}, t)[33]$.

When the system passes through the nonadiabatic coupling region, the electron transfers probability between the first excited state and the ground state (top panel of Fig. 4). As a result of the electronic transitions, the reduced nuclear density changes shape by splitting into two parts representing influences from both ground and excited state BOPES's at $t \approx 32$ fs (middle panel of Fig. 4). As nonadiabatic transitions occur, the system builds up a degree of coherence, and this coherence subsequently decays as the system evolves away from the coupling region (bottom panel of Fig. 4). The interacting-CWF method vastly outperforms the HermitianCWF approach [26], Ehrenfest mean field theory, as well as Tully's surface hopping dynamics (also shown in Fig. 4) [39] in describing all these aspects of this problem. While both the interacting-CWF method and Ehrenfest dynamics correctly capture the exact population dynamics at short times, the latter breaks down at longer times. Mean field theory also fails to capture the qualitative structure of the time-evolving reduced nuclear density, and the indicator of decoherence. These features are perfectly captured by our interacting-CWF approach using very few trajectories; fully converged interacting-CWF results were reached with 250 trajectories, while $2 \times 10^{4}$ trajectories (initially sampled from the Wigner distribution corresponding to the initial quantum nuclear wave packet) were required for convergence with Ehrenfest dynamics. Somewhat surprisingly, as shown in the bottom panel of Fig. 1, electronic decoherence is captured nearly quantitatively by the interacting-CWF method using only 10 trajectories.

\section{B. Nonequilibrium dynamics in a cavity bound electron-photon system}

Next, we simulate a single electron in a one-dimensional double well potential that is coupled to a quantum electrodynamical (QED) cavity through a single photon mode in the ultrastrong-coupling regime [40-42] (see Fig. 5). The Hamiltonian for the coupled electron-photon system is given

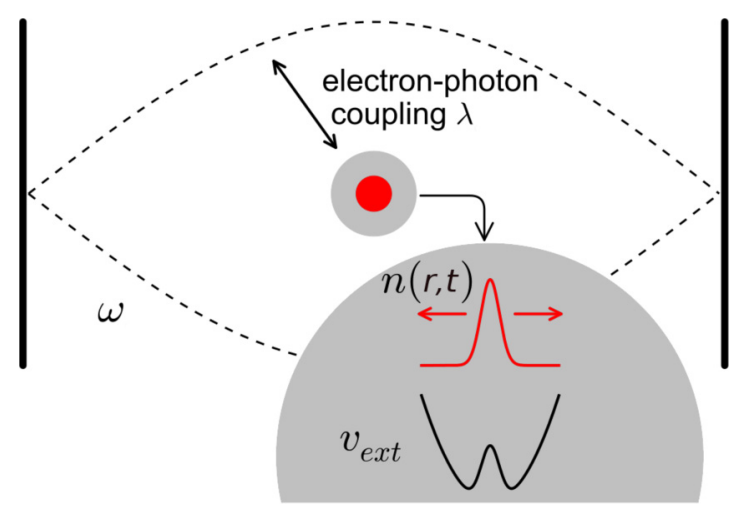

FIG. 5. The figure schematically illustrates a 1D optical cavity containing one atom, with a single electron. The coupling of the electron to the cavity mode at resonance frequency $\omega$ and with electron-photon coupling strength $\lambda$ modifies the dynamics of the electron density $n(r, t)$, which moves in a double-well potential represented by $v_{\text {ext }}$. 


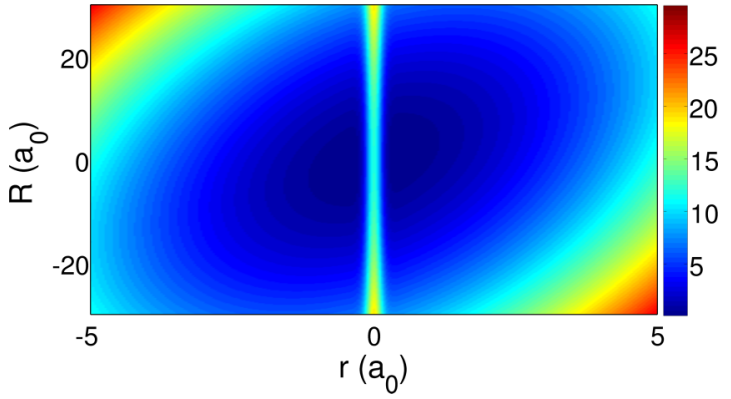

FIG. 6. Full electron-photon potential $\hat{W}(r, R)$ for the model system used in this work (depicted in Fig. 5).

by the following expression [40]:

$$
\hat{H}(r, R)=-\frac{1}{2 m} \frac{\partial^{2}}{\partial r^{2}}-\frac{1}{2 M} \frac{\partial^{2}}{\partial R^{2}}+\hat{W}(r, R),
$$

where $m$ and $M$ are the electron mass and the effective mass of the mode of the quantized displacement field.

The first two terms of the Hamiltonian describe the nonrelativistic kinetic energy of the electron and the kinetic energy operator of the quantized displacement-field component for a single photonic mode (with $M=1$ in atomic units). The full electron-photon potential is depicted in Fig. 6 and reads

$$
\hat{W}(r, R)=\frac{\omega^{2}}{2}\left(R-\frac{\lambda}{\omega} r\right)^{2}+\frac{\Omega}{2} r^{2}+V_{o} e^{-r^{2} / d^{2}} .
$$

The electron-photon interaction is described in the dipole approximation, i.e., the dipole-moment operator $r$ couples linearly to the photon displacement coordinate $R$, which is proportional to the quantized displacement field component of the mode, i.e., $D \propto \omega \lambda R$. The value of the electron-photon coupling $\lambda$ can be changed from the weak- to the ultrastrongcoupling limit in circuit QED experiments [41,42]. In addition, the Hamiltonian contains a quadratic electron selfinteraction $v_{\mathrm{es}}=\frac{\lambda^{2} r^{2}}{2}$ and an external potential $v_{\mathrm{ext}}=\frac{\Omega}{2} r^{2}+$ $V_{o} e^{-r^{2} / d^{2}}$ that introduces an additional confining potential plus a quantum barrier that forces the electron to tunnel as it couples to the photonic mode.

The parameter regime studied in this model is defined by the parameters $\Omega=0.7827, V_{0}=12, d=0.15, \omega=0.1249$.

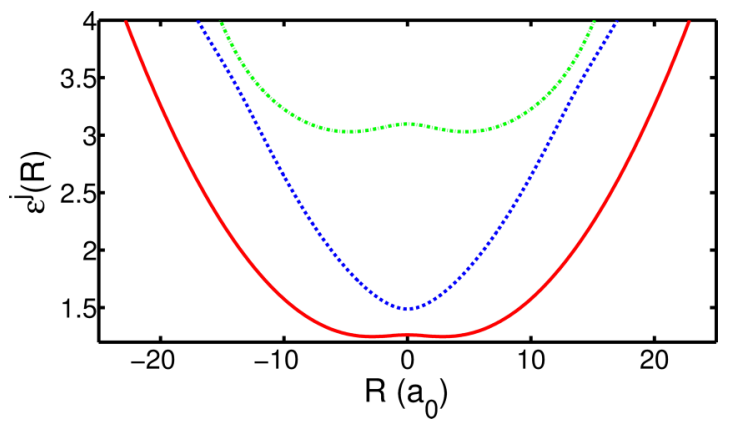

FIG. 7. BOPESs for the electron-photon model system used in this work. Ground state, $\epsilon^{(1)}$, first excited state, $\epsilon^{(2)}$, and second excited state, $\epsilon^{(3)}$, BOPESs are shown, respectively, by a red solid line, a blue dashed line, and a green dotted line.
Furthermore, we chose the electron-photon coupling to be $\lambda=0.4$, which corresponds to the ultrastrong-coupling regime. This scenario constitutes a formidable challenge for approximate approaches. The ground Born-Oppenheimer potential-energy surface (BOPES), $\epsilon^{(1)}$, is strongly coupled to the first excited adiabatic state, $\epsilon^{(2)}$, around $R_{12}=0 a_{0}$. There is also a non-negligible coupling between the first excited and second adiabatic states at around $R_{23}=-5 a_{0}$ and $R_{23}=5 a_{0}$. The coupling to the rest of the BOPESs is negligible (see Fig. 7). The effective mass of the photon displacement coordinate is identical to the electronic mass, hence the dynamics deviates strongly from the BO limit. Furthermore, tunneling, quantum coherence, and zero-point energy conservation are also important for both interacting subsystems.
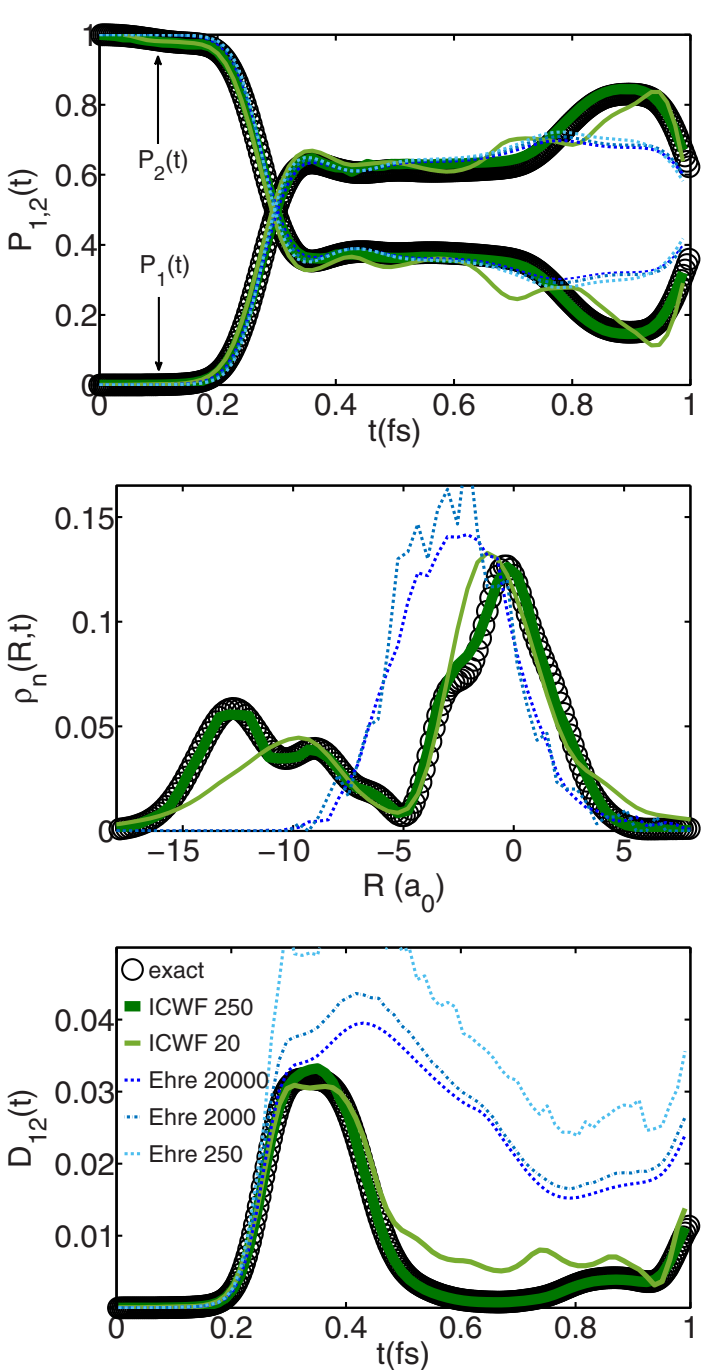

FIG. 8. Nonequilibrium QED cavity-bound electronic dynamics in the ultrastrong-coupling regime. Exact (black circles) and interacting-CWF with $M=250$ (dark green line) and $M=20$ (light green line), and Ehrenfest mean field theory results with $M=20000$ (dark blue dashed line), $M=2000$ (blue dashed line), and $M=250$ (light blue dashed line). Top panel: evolution of electronic BO state populations. Middle panel: reduced quantized displacement-field coordinate probability density $\rho(R, t)$ at $t=1$ fs. Bottom panel: time-dependent decoherence indicator $D_{12}(t)$. 
We choose the system to be initially prepared with the electron in the first excited BO state, $\epsilon^{(2)}$, while the initial "photonic" wave function is prepared in a displaced coherent state, with $\sigma=1 / \sqrt{0.05}$, centered at $R=-10.0 a_{0}$. The time-step used for integrating the TDSE was chosen to be $2.4 \times 10^{-5}$ fs (or $0.001 a_{0}$ ). The fourth-order Runge-Kutta algorithm is used to propagate the CWF equations of motion and the corresponding trajectories, and we again used the Moore-Penrose pseudoinversion method in propagating the time-dependent coefficients.

This initial state described above is not a stationary eigenstate of the full electron-photon Hamiltonian, and it evolves in time by scattering thorough multiple avoided crossings. The reduced photon density develops a complex structure as time progresses (middle panel of Fig. 8). The exact electronic and photonic dynamics are quantitatively captured by the interacting-CWF approach, as shown in Fig. 8, again with an extremely small trajectory ensemble. Again Ehrenfest dynamics correctly captures the exact population dynamics at short times, but breaks down at long times. Mean field theory also fails to capture the qualitative structure of the time-evolving reduced nuclear density and hence the indicator of decoherence.

\section{CONCLUSIONS}

To summarize, we presented a method for solving the TDSE that is based on the recently introduced, exact, conditional decomposition of the many-body wave function [26]. We use the lowest order solution to the CWF equations of motion as a time-dependent basis, in a stochastic wavefunction ansatz that we call the interacting-CWF approach. Our simulation results for the coupled electron-nuclear and electron-photon model system show that this method captures a quantitatively accurate physical picture, while using a number of trajectories that is orders of magnitude lower than the corresponding mean-field simulation. The degree of computational efficiency offered by this approach creates the possibility to treat dynamics in molecular and extended quantum systems with unprecedented accuracy without the need to precompute the BOPESs or NACTs, while providing access to all observables relevant for describing nonequilibrium dynamical phenomena.

In addition, these developments provide a general framework to approach the many-body problem in a variety of contexts. Notice that the decomposition of the full wave function offered here, in (3) and (4), is but one option of many possible conditional decompositions of the interacting manybody wave function. For example, using single-particle CWFs in a form compatible with time-dependent density functional theory is another particularly appealing route to follow in this respect, and work in this direction is already in progress.

\section{ACKNOWLEDGMENTS}

All of the authors thank Shunsuke Sato, Heiko Appel, and Tarek A. Elsayed for fruitful discussions. G.A. acknowledges financial support from the European Unions Horizon 2020 research and innovation programme under the Marie Skodowska-Curie Grant Agreement No. 752822, the Spanish Ministerio de Economa y Competitividad (Project No. CTQ2016-76423-P), and the Generalitat de Catalunya (Project No. 2017 SGR 348). A.K. acknowledges funding from the National Sciences and Engineering Research Council of Canada Discovery grant program. A.R. acknowledges financial support from the European Research Council (Grant No. ERC-2015-AdG-694097) and Grupos Consolidados (Project No. IT578-13).
[1] W. H. Miller, J. Chem. Phys. 136, 210901 (2012).

[2] J. C. Tully, J. Chem. Phys. 137, 22A301 (2012).

[3] R. Kapral, J. Phys.: Condens. Matter 27, 073201 (2015).

[4] A. McLachlan, Mol. Phys. 8, 39 (1964).

[5] J. C. Tully, J. Chem. Phys. 93, 1061 (1990).

[6] T. J. Martinez, M. Ben-Nun, and R. Levine, J. Phys. Chem. 100, 7884 (1996).

[7] G. A. Worth and I. Burghardt, Chem. Phys. Lett. 368, 502 (2003).

[8] G. Worth, M. Robb, and I. Burghardt, Faraday Discuss. 127, 307 (2004)

[9] D. V. Shalashilin, J. Chem. Phys. 132, 244111 (2010).

[10] D. V. Makhov, W. J. Glover, T. J. Martinez, and D. V. Shalashilin, J. Chem. Phys. 141, 054110 (2014).

[11] X. Sun and W. H. Miller, J. Chem. Phys. 106, 6346 (1997).

[12] W. H. Miller, J. Phys. Chem. A 113, 1405 (2009).

[13] R. Kapral and G. Ciccotti, J. Chem. Phys. 110, 8919 (1999).

[14] A. Kelly, R. van Zon, J. Schofield, and R. Kapral, J. Chem. Phys. 136, 084101 (2012).

[15] C.-Y. Hsieh and R. Kapral, J. Chem. Phys. 137, 22A507 (2012).

[16] Q. Shi and E. Geva, J. Chem. Phys. 118, 8173 (2003).

[17] S. Bonella and D. Coker, J. Chem. Phys. 122, 194102 (2005).
[18] E. Dunkel, S. Bonella, and D. Coker, J. Chem. Phys. 129, 114106 (2008).

[19] P. Huo and D. F. Coker, J. Chem. Phys. 137, 22 A535 (2012).

[20] A. Abedi, N. T. Maitra, and E. K. U. Gross, Phys. Rev. Lett. 105, 123002 (2010).

[21] S. K. Min, F. Agostini, and E. K. U. Gross, Phys. Rev. Lett. 115 , 073001 (2015).

[22] J.-K. Ha, I. S. Lee, and S. K. Min, J. Phys. Chem. Lett. 9, 1097 (2018).

[23] M. Born and R. Oppenheimer, Ann. Phys. 389, 457 (1927).

[24] W. Domcke and G. Stock, Theory of Ultrafast Nonadiabatic Excited-State Processes and their Spectroscopic Detection in Real Time (Wiley, New York, 2007), pp. 1-169.

[25] G. Albareda, A. Abedi, I. Tavernelli, and A. Rubio, Phys. Rev. A 94, 062511 (2016).

[26] G. Albareda, H. Appel, I. Franco, A. Abedi, and A. Rubio, Phys. Rev. Lett. 113, 083003 (2014).

[27] D. Bohm, Phys. Rev. 85, 166 (1952).

[28] E. Gindensperger, C. Meier, and J. Beswick, J. Chem. Phys. 113, 9369 (2000).

[29] B. F. E. Curchod, I. Tavernelli, and U. Rothlisberger, Phys. Chem. Chem. Phys. 13, 3231 (2011). 
[30] B. Gu and S. Garashchuk, J. Phys. Chem. A 120, 3023 (2016).

[31] G. Albareda, J. M. Bofill, I. Tavernelli, F. Huarte-Larrañaga, F. Illas, and A. Rubio, J. Phys. Chem. Lett. 6, 1529 (2015).

[32] T. A. Elsayed, K. Mølmer, and L. Bojer Madsen, Sci. Rep. 8, 12704 (2018).

[33] See Supplemental Material at http://link.aps.org/supplemental/ 10.1103/PhysRevMaterials.3.023803 for a detailed description of the numerical implementation of the ICWF method including specific expressions to compute the electronic BO populations, the reduced nuclear coordinate density, and the decoherence indicator in terms of the ansatz in (7).

[34] A. Benseny, G. Albareda, Á. S. Sanz, J. Mompart, and X. Oriols, Eur. Phys. J. D 68, 286 (2014).
[35] X. Oriols, Phys. Rev. Lett. 98, 066803 (2007).

[36] M. Beck, A. Jäckle, G. Worth, and H.-D. Meyer, Phys. Rep. 324, 1 (2000).

[37] S. Shin and H. Metiu, J. Chem. Phys. 102, 9285 (1995).

[38] A. Abedi, F. Agostini, Y. Suzuki, and E. K. U. Gross, Phys. Rev. Lett. 110, 263001 (2013).

[39] F. Agostini, A. Abedi, Y. Suzuki, S. K. Min, N. T. Maitra, and E. K. U. Gross, J. Chem. Phys. 142, 084303 (2015).

[40] J. Flick, M. Ruggenthaler, H. Appel, and A. Rubio, Proc. Natl. Acad. Sci. USA 112, 15285 (2015).

[41] J. Flick, M. Ruggenthaler, H. Appel, and A. Rubio, Proc. Natl. Acad. Sci. USA 114, 3026 (2017).

[42] M. Ruggenthaler, N. Tancogne-Dejean, J. Flick, H. Appel, and A. Rubio, Nat. Rev. Chem. 2, 0118 (2018). 